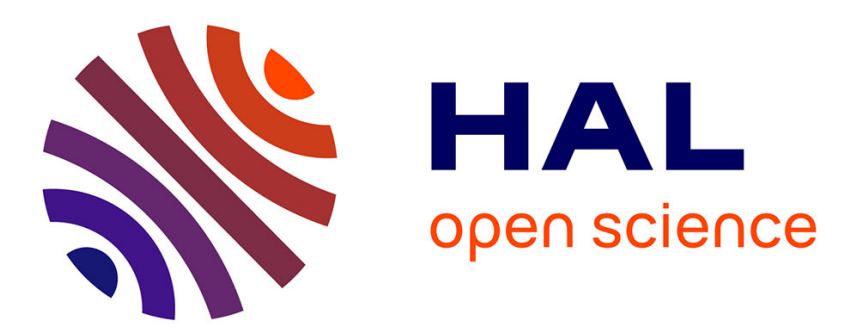

\title{
Determination of a reaction scheme for cardboard thermal degradation using thermal gravimetric analysis
}

Céline David, Sylvain Salvador, Jean-Louis Dirion, Michel Quintard

\section{To cite this version:}

Céline David, Sylvain Salvador, Jean-Louis Dirion, Michel Quintard. Determination of a reaction scheme for cardboard thermal degradation using thermal gravimetric analysis. Journal of Analytical and Applied Pyrolysis, 2003, 67 (2), pp.307-323. 10.1016/S0165-2370(02)00070-0 . hal-01718083

\section{HAL Id: hal-01718083 \\ https://hal.science/hal-01718083}

Submitted on 7 Nov 2018

HAL is a multi-disciplinary open access archive for the deposit and dissemination of scientific research documents, whether they are published or not. The documents may come from teaching and research institutions in France or abroad, or from public or private research centers.
L'archive ouverte pluridisciplinaire HAL, est destinée au dépôt et à la diffusion de documents scientifiques de niveau recherche, publiés ou non, émanant des établissements d'enseignement et de recherche français ou étrangers, des laboratoires publics ou privés. 


\title{
Determination of a reaction scheme for cardboard thermal degradation using thermal gravimetric analysis
}

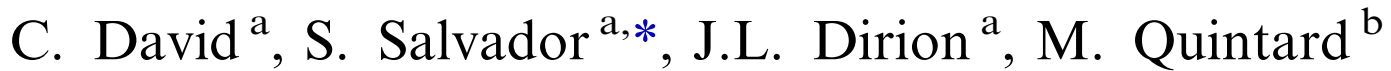 \\ a'École des Mines d'Albi-Carmaux-UMR 2392 CNRS, Campus Jarlard, route de Teillet, 81013 Albi CT \\ Cédex 09, France \\ ${ }^{\mathrm{b}}$ Institut de Mécanique des Fluides de Toulouse-UMR 5502 CNRS, Allée du Pr Camille Soula, 31400 \\ Toulouse, France
}

\begin{abstract}
In this paper, we present new thermogravimetic analysis on cardboard material performed at different heating rates. Several reaction schemes are proposed to serve as an interpretation basis. Considering the experiments independently, it is found that the best fitted parameters are highly sensitive upon the heating rate. In order to avoid complicated and hazardous interpolation schemes, a simplest interpretation is proposed that does not involve a heating rate dependency on the kinetic parameters.
\end{abstract}

Keywords: Thermal gravimetric analysis; Cardboard; Pyrolysis; Reaction scheme; Municipal solid waste

\section{Introduction}

Cardboard is largely used today for food, equipment, and various packaging. This material represents about $30 \%$ of municipal solid waste, a proportion increasing every year. Recycling is sometimes not possible, either when sorting is difficult or

* Corresponding author. Tel.: +33-5-6349-3127; fax: +33-5-6349-3099

E-mail address: salvador@enstimac.fr (S. Salvador). 
when the material is too dirty. The high heating value $\left(\approx 15500 \mathrm{~kJ} \mathrm{~kg}^{-1}\right)$ and the low pollutant emissions during its combustion make thermal valorization an interesting perspective. The first physical phenomenon governing the combustion of such solid materials is the devolatilization, a step during which cardboard produces gas, to end with char, a solid residue mainly composed of carbon. This step corresponds to pyrolysis since the reaction can operate under inert atmosphere. The objective of this paper is to discuss this pyrolysis phenomenon on the basis of a series of TGA experiments.

Cardboard is a complex material, essentially composed of cellulose, hemicellulose and lignin. Only few studies have been devoted to the kinetics of its pyrolysis. The reaction schemes found in the literature are 'apparent' schemes: they describe the evolution of the total solid mass of a sample. Gupta [1] proposed a two steps reaction scheme, with different sets of Arrhenius parameters depending on the heating rate. This scheme was suggested by the shape of the mass loss curves, in which two regions with very different mass loss rates are clearly identified.

Because of a similar chemical composition, we may consider that cardboard behavior during pyrolysis is similar to that of paper [1,2], wood [3], or cellulose [4-9], to which numerous articles were devoted. In the case of cellulose pyrolysis, an extensive literature exists on the subject, we refer the reader to the review paper by Antal [7] for more information. For this type of material, different reaction schemes have been proposed. Among the most often used, we can note the Broido and Nelson model [10] in which two competitive reactions convert cellulose into volatile tars, char and volatile components (i.e. compounds with a low molecular weight). This reaction scheme can be schematically represented as

$$
\begin{aligned}
& \text { Cellulose } \stackrel{k_{1}}{\rightarrow} \text { volatile tars } \\
& \text { Cellulose } \stackrel{k_{2}}{\rightarrow} \text { char }+ \text { volatiles }
\end{aligned}
$$

In the model proposed by Bradbury et al. [11], cellulose is first converted into active cellulose, which is then converted by two competitive reactions into volatile comonents, char and gas. The successive reactions can be represented as

$$
\begin{aligned}
& \text { Cellulose } \stackrel{k_{1}}{\rightarrow} \text { active cellulose } \underset{k_{3}}{\stackrel{k_{2}}{\rightarrow}} \text { volatiles tars } \\
& \text { active cellulose } \stackrel{k_{3}}{\rightarrow} \text { char }+ \text { gas }
\end{aligned}
$$

In the case of flash pyrolysis of cellulose, Hajaligol [4,5] proposed the following reaction scheme, involving a large number of reactions, each reaction converting cellulose into a different component:

$$
\text { Cellulose } \stackrel{k_{i}}{\rightarrow} \text { Product } i
$$

Concerning paper devolatilization, a model with two successive reactions has been tested by Gupta [1] and $\mathrm{Wu}$ [2]. The reaction scheme is the following, in which 'Intermediate' is a solid pseudo-species: 


\section{Paper $\stackrel{k_{1}}{\rightarrow}$ Intermediate + Gases}

$$
\text { Intermediate } \stackrel{k_{2}}{\rightarrow} \text { Char }+ \text { Gases }
$$

Besides the consistency with the idea of a complex cardboard chemical composition, the reason why authors develop models involving several reactions is that one can observe differences in the gas production and the solid residue yield, depending on the heating rate that the sample undergoes. A single reaction model cannot predict such a behavior. In particular, this was demonstrated in the case of cellulose [7,9], and also in the case of paper [1,2]. This effect was taken into account, as proposed by the authors, by determining a different set of Arrhenius parameters for each experiment performed at a different heating rate. In practice, however, there is no particular reason for having a constant heating rate. Modeling such variable situations would require the introduction of complex, non-linear dependence of the reaction parameters, with respect to the actual heating rates. This may lead to intricate complications as explained below.

The modeling of thermal degradation of a large solid body (particles, bricks, materials in a fixed bed) generally requires solving heat and mass balance equations to compute the temperature and the gas composition at each location inside the material. The heat and mass source terms are calculated from the chemical reactions progress, which is itself computed from a reaction scheme involving the Arrhenius parameters. As discussed before, the heating rate (HR) is a parameter that largely impacts on the chemical reaction progress and on the species production. Since the heating rate varies from one location to another inside the large solid body, the Arrhenius parameters should be clearly expressed for any case. The kinetic parameters can be determined for a number of discrete HR. If one requires the values of the Arrhenius parameters for any HR, they must be interpolated from the discrete values, for instance by using a non-linear interpolation function. As it will be demonstrated later in this paper, this operation is delicate, and likely to introduce some error.

As an alternate route, this paper is an attempt to derive a single reaction scheme able to predict reasonably the sample mass evolution undergone by cardboard whatever the heating rate (in a specified range). The results are based on a series of new thermogravimetric (TG) experiments performed at three HR: 3, 10 and $50 \mathrm{~K}$ $\min ^{-1}$. These values cover a large range of practical cases, while it must be acknowledged that larger heating rates can be locally encountered, for example in the cases of small particles or for the area very closed to the surface of large solid bodies submitted to a high heat flux. Several reaction schemes are proposed. For each scheme, the corresponding Arrhenius parameters and stoechiometric coefficients are determined by fitting the total sample mass evolution curves predicted by the model, at the three HR, with the corresponding curves for the three experiments. We a priori expect to gain some information in terms of the chemical mechanism sequence, postulating that the more representative reaction scheme should lead to the best fit with experimental results. 


\section{Experimental results}

Thermal decomposition of cardboard was realized in an inert environment (nitrogen) using a Setaram ${ }^{\mathrm{TM}}$ 92-16.18 thermo-gravimetric analyser (TGA). The general classical arrangement of a TGA apparatus is sketched in Fig. 1. The recorded signals are the sample mass and temperature. This last parameter is measured at the crucible bottom, in order to minimize the temperature difference between the thermocouple and the sample during dynamic tests.

Cardboard samples were finely pulverized by cryogenic grinding. The initial mass $7-15 \mathrm{mg}$ is subjected during the test to the following procedure. First, a vacuum purge is realized to eliminate oxygen. Then, to stabilize the system, the temperature of $293 \mathrm{~K}$ is maintained during $20 \mathrm{~min}$. The sample is then heated progressively from 293 to $873 \mathrm{~K}$. Three experiments were performed with heating rates of 3,10 and $50 \mathrm{~K}$ $\min ^{-1}$.

The obtained mass loss curves (normalized by the initial mass) are represented in Fig. 2. They have all the same shape. A first mass loss of approximately $2.5 \%$ of the initial mass occurs between 313 and $450 \mathrm{~K}$. It is attributed to drying. The difference in the drying mass loss between the three tests may only depend on the initial natural water content of the cardboard samples. The cardboard thermal decomposition that occurs later causes a more important mass loss. It apparently occurs in two steps: a first, rapid one for temperatures up to $630-680 \mathrm{~K}$, and a second, slower one at higher temperatures. This thermal decomposition (between 500 and $873 \mathrm{~K}$ ) lasts approximately for 120,37 and 7.5 min for the experiments at HR equal to 3,10 and $50 \mathrm{~K} \mathrm{~min}^{-1}$, respectively. A stable mass was obtained after waiting for $1 \mathrm{~h}$ at $873 \mathrm{~K}$; the corresponding asymptotic value is represented on Fig. 2. Each experiment has been performed two to three times in order to check the repeatability of the mass loss curves. When the mass is normalized to the dry product (to get rid of the drying

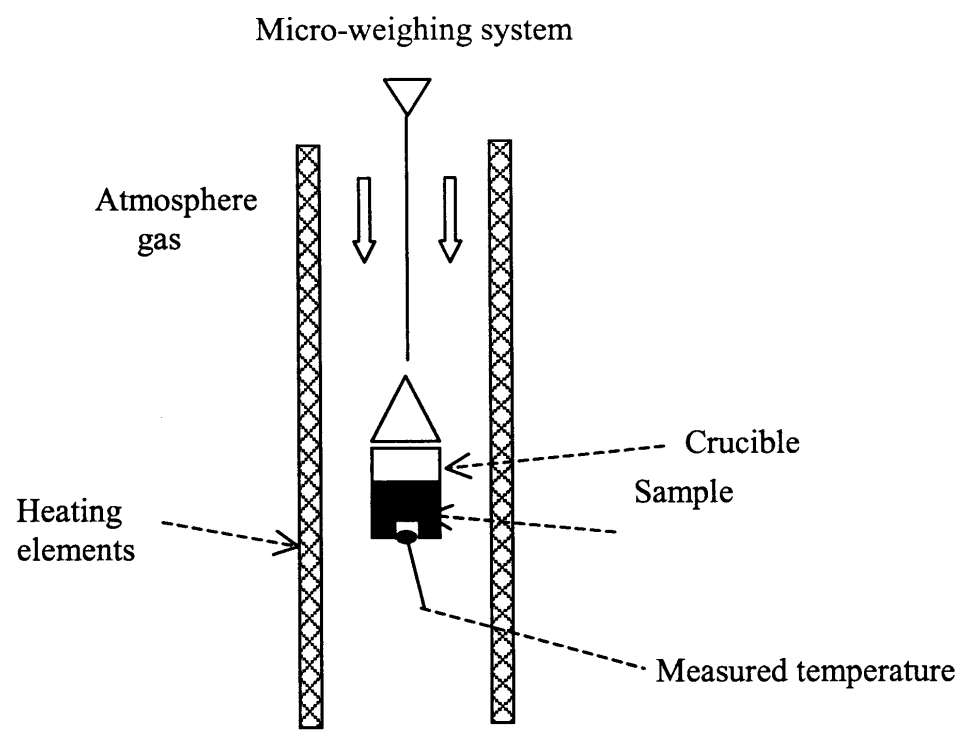

Fig. 1. Principle of the TGA apparatus. 


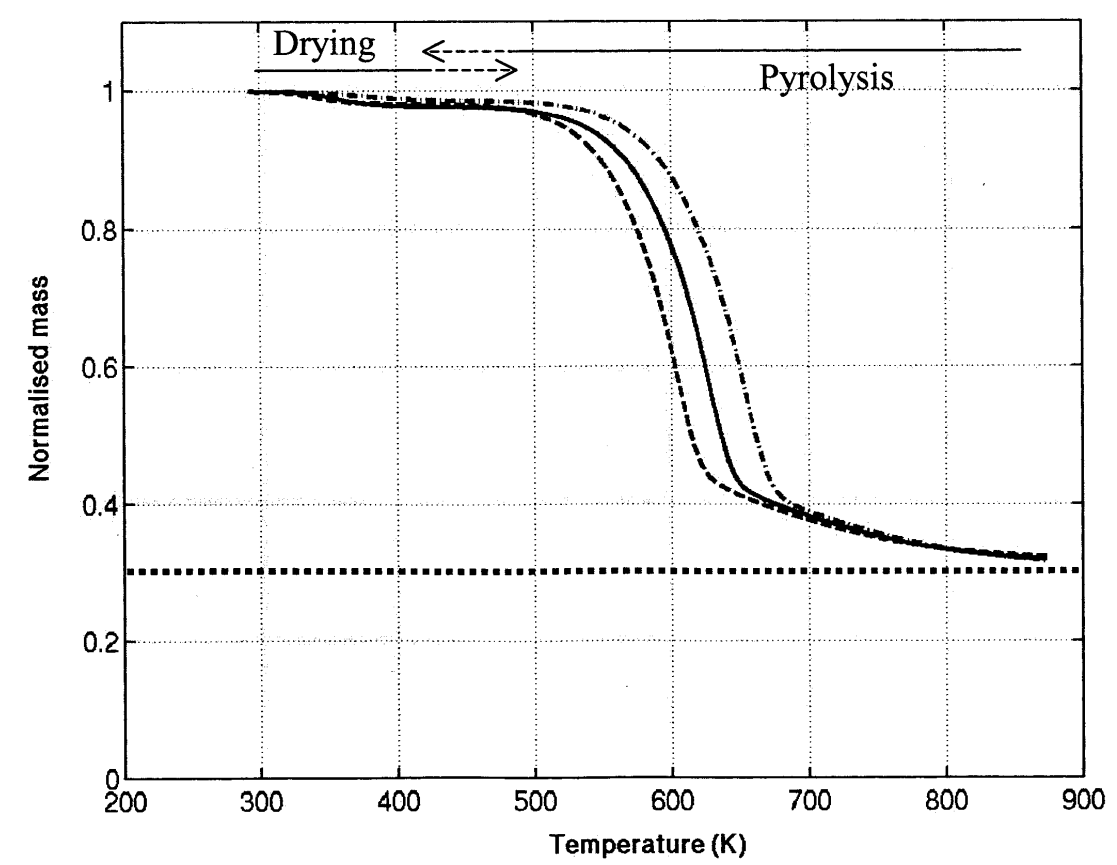

Fig. 2. Normalized mass evolution vs. temperature at three different heating rates: (- -) $3 \mathrm{~K} \mathrm{~min}^{-1}$, (-) 10 $\mathrm{K} \min ^{-1}$ and (- .) $50 \mathrm{~K} \mathrm{~min}^{-1}$.

stage) mass differences less than $1 \%$ were obtained, which indicates a good reproducibility of the experiments.

A more detailed interpretation can be done using an Arrhenius representation of the apparent reaction rates. The total mass loss kinetics is interpreted using the following model

$$
\frac{\mathrm{d} m}{\mathrm{~d} t}=-k m
$$

where $m$ is the solid mass that can react (excluding moisture water). Fig. 3 represents the evolution of the reaction rate $k$ versus $1 / T$ for the three different heating rates. The curves exhibit two temperature domains where the plots are almost linear. An interpretation could be that each linear domain corresponds to a specific single reaction, such as the decomposition of two different chemical species. However, the reaction rates for a given temperature are very different depending on the heating rate. Indeed, a ratio of 20 can be reached between the highest and the lowest value. A possible explanation of this phenomenon is that the observed global mass loss is the sum of mass losses due to different reactions occurring simultaneously, involving different masses for the different species.

It is also surprising that these differences in the apparent reaction rate for the experiments at different HRs are present at the very beginning of the pyrolysis process, i.e. $1.8 \times 10^{-3}<1 / T<2.2 \times 10^{-3}$. It should nevertheless be kept in mind that these values were calculated from very small values of the mass derivatives $(\mathrm{d} m /$ $\mathrm{d} t$ ), thus resulting in a loss of accuracy. For clarity, we have hatched for each curve 


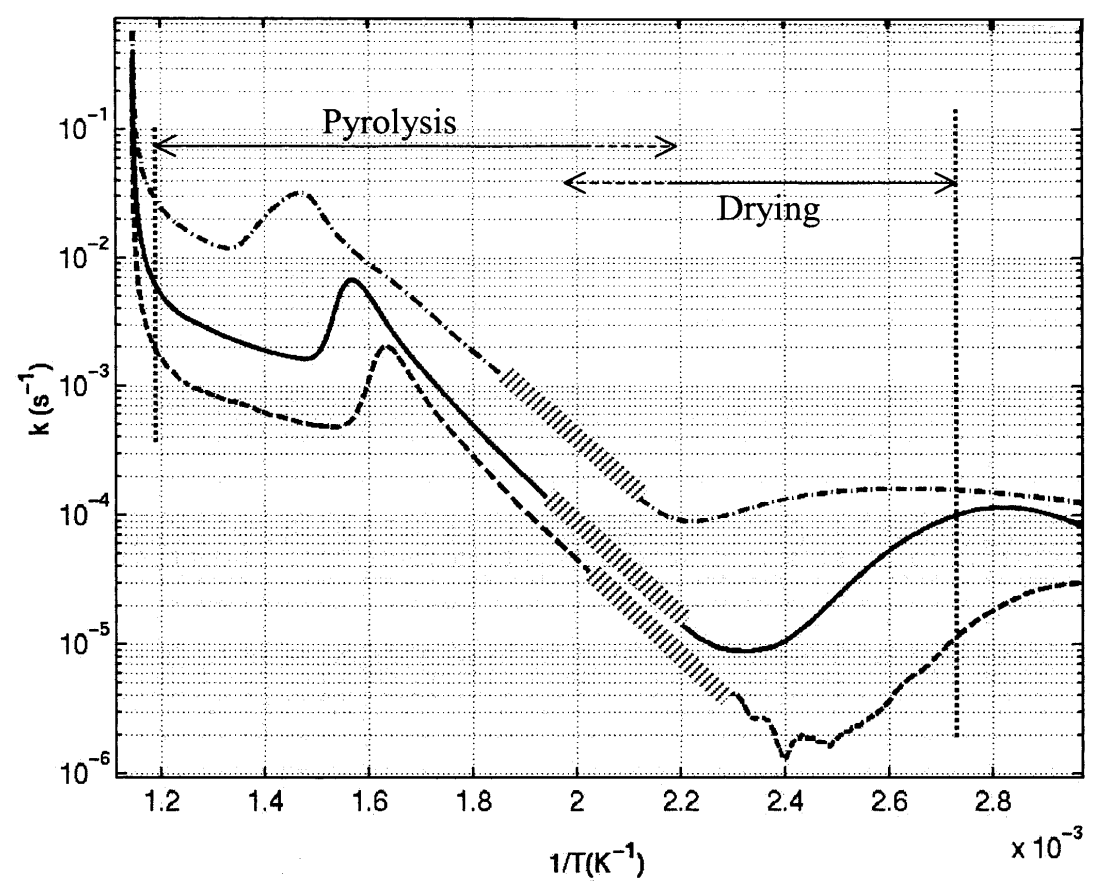

Fig. 3. Evolution of $k$ vs. $1 / T$ for experiments at three different heating rates: (- -) $3 \mathrm{~K} \mathrm{~min}^{-1}$, (-) $10 \mathrm{~K}$ $\min ^{-1}$ and (- .) $50 \mathrm{~K} \mathrm{~min}^{-1}$.

the zone corresponding to a mass loss of less than $2 \%$, zones to which little attention should be paid.

From this analysis, it appears that the thermal degradation of cardboard is a complex sum of different reactions, some of them occurring simultaneously. It is beyond the scope of this paper to propose reaction schemes for cardboard pyrolysis in terms of real reacting and produced chemical species. Instead, given the data at our disposal, we will propose an approximation by testing schemes with several simple reactions with the objective to reproduce the mass evolution whatever the applied heating rate. Each reaction of a given scheme will consider pseudo-species as detailed below.

\section{Reactions schemes}

The mass evolution of a constituent undergoing a single reaction is traditionally described as

$$
\frac{\mathrm{d} m}{\mathrm{~d} t}=-k m^{n}
$$

where $m$ is the reactive sample mass, $t$ is time and $k$ the reaction rate. The reaction order $n$ is most of the time taken equal to one [12]. Choosing a value different from 1 implies mathematically that the initial mass has an impact on the mass loss rate, a phenomenon that can physically only be attributed to mass transfer effects. All the reactions considered below will therefore be written with $n=1$. 
The reaction rate, $k$, is determined with the Arrhenius law

$$
k=A \exp \left(-\frac{E a}{R T}\right)
$$

where $A$ (pre-exponential factor) and $E a$ (activation energy) are the Arrhenius parameters, $R$ is the universal gas constant and $T$ is the temperature in Kelvin.

The reaction schemes we have tested are presented in Table 1. All of them are simple classical mechanisms, describing the global mass loss as the sum of mass losses due to gas release during a number of parallel or consecutive reactions. These reactions involve the following pseudo-species: cardboard $(\mathrm{C})$, intermediate species (Ii), char (Char) and gas (G). Char is supposed to be a stable chemical species (like carbon). The coefficients $a, b, c, d, a^{\prime}, b^{\prime}, c^{\prime}$ and $d^{\prime}$ are stoechiometric mass coefficients. The chemical significance of these reaction schemes is discussed below.

Scheme \#1 assumes that cardboard (C) produces an intermediate species (I1) and gas. This intermediate species is eventually converted into char and gas through a second reaction. We consider in Scheme \#2 the case where char is produced through two successive intermediate species (I1 and I2). In Scheme \#3, two different intermediate species are produced simultaneously by the same reaction. The proportion between these species depend on the stoechiometric coefficients $a$ and $b$. The two intermediate species are subsequently converted into char and gas through two different reactions. Schemes \#4 and \#5 postulate that cardboard is converted into different species by two competitive reactions; the solid intermediate species are then converted into char and gas. The possible initial presence of two

Table 1

Reaction schemes considered in this work for cardboard thermal degradation

\begin{tabular}{|c|c|c|}
\hline$\#$ & Reactions scheme description & searched parameters \\
\hline 1 & $\begin{array}{l}C \stackrel{k_{1}}{\rightarrow} a \mathrm{I} 1+a^{\prime} G \\
a \mathrm{I} 1 \stackrel{k_{2}}{\rightarrow} b \text { Char }+b^{\prime} G\end{array}$ & $\begin{array}{l}A_{1}, E a_{1}, a \\
A_{2}, E a_{2}, b\end{array}$ \\
\hline 2 & $\begin{array}{l}C \stackrel{k_{1}}{\rightarrow} a \mathrm{I} 1+a^{\prime} G \\
a \mathrm{I} 1 \stackrel{k_{2}}{\rightarrow} b \mathrm{I} 2+b^{\prime} G \\
b \mathrm{I} 2 \stackrel{k_{3}}{\rightarrow} c \mathrm{Char}+c^{\prime} G\end{array}$ & $\begin{array}{l}A_{1}, E a_{1}, a \\
A_{2}, E a_{2}, b \\
A_{3}, E a_{3}, c\end{array}$ \\
\hline 3 & $\begin{array}{l}C \stackrel{k_{1}}{\rightarrow} a \mathrm{I} 1+b \mathrm{I} 2+a^{\prime} G \\
a \mathrm{I} 1 \underset{k_{2}}{\stackrel{k_{3}}{\rightarrow}} c \text { Char }+c^{\prime} G \\
b \mathrm{I} 2 \underset{k_{1}}{\rightarrow} d \mathrm{Char}+d^{\prime} G\end{array}$ & $\begin{array}{l}A_{1}, E a_{1}, a, b \\
A_{2}, E a_{2}, c \\
A_{3}, E a_{3}, d\end{array}$ \\
\hline 4 & $\begin{array}{l}C \stackrel{k_{1}}{\rightarrow} a \mathrm{I} 1+a^{\prime} G \\
C \stackrel{k_{2}}{\rightarrow} b \mathrm{Char}+b^{\prime} G \\
a \mathrm{I} 1 \stackrel{k_{3}}{\rightarrow} c \text { Char }+c^{\prime} G\end{array}$ & $\begin{array}{l}A_{1}, E a_{1}, a \\
A_{2}, E a_{2}, b \\
A_{3}, E a_{3}, c\end{array}$ \\
\hline 5 & $\begin{array}{l}\alpha C 1 \stackrel{k_{1}}{\rightarrow} a \mathrm{I} 1+a^{\prime} G \\
(1-\alpha) C 2 \stackrel{k_{2}}{\rightarrow} b \text { Char }+b^{\prime} G \\
a \mathrm{I} 1 \stackrel{k_{3}}{\rightarrow} c \text { Char }+c^{\prime} G\end{array}$ & $\begin{array}{l}A_{1}, E a_{1}, a, \alpha \\
A_{2}, E a_{2}, b \\
A_{3}, E a_{3}, c\end{array}$ \\
\hline 6 & $\begin{array}{l}C \stackrel{k_{1}}{\rightarrow} a \mathrm{I} 1+a^{\prime} G \\
C \stackrel{k_{2}}{\rightarrow} b \mathrm{I} 2+b^{\prime} G \\
a \mathrm{I} 1 \stackrel{k_{3}}{\rightarrow} c \text { Char }+c^{\prime} G \\
b \mathrm{I} 2 \stackrel{k_{4}}{\rightarrow} d \mathrm{Char}+d^{\prime} G\end{array}$ & $\begin{array}{l}A_{1}, E a_{1}, a \\
A_{2}, E a_{2}, b \\
A_{3}, E a_{3}, c \\
A_{4}, E a_{4}, d\end{array}$ \\
\hline
\end{tabular}


different species (denoted $\mathrm{C} 1$ and $\mathrm{C} 2$ ) in the cardboard material is considered in Scheme \#5. This offers the possibility to describe the cardboard thermal degradation as the parallel degradation of two compounds initially present in the material, for instance cellulose and lignin. The proportion between $\mathrm{C} 1$ and $\mathrm{C} 2$ is determined by the coefficient $\alpha$. The first of these two species is converted into an intermediate species and gas; the second is converted into char and gas. In Scheme \#6, cardboard is converted into two intermediate species by two competitive reactions. The two intermediate compounds are supposed to have different thermochemical properties, resulting in different char productions, following different reaction kinetics.

It is noteworthy that for all the schemes, the future identification of all the stoechiometric coefficients leaves the possibility to set one (or several) of them to zero, which increases the degrees of freedom of the schemes.

\section{Arrhenius parameters determination}

Once a kinetic scheme has been selected, two main methods can be used to estimate the corresponding Arrhenius parameters $A$ and $E a$ : a graphical method or numerical fitting.

\subsection{Graphical method}

From the values of $m$ and $\mathrm{d} m / \mathrm{d} t$ obtained from the TGA experiments, we can calculate $k$ (Eq. (5)) for all the temperature range explored in the experiments [13]. From the expression of $k$ (Eq. (7)), we have:

$$
\ln k=\ln A-\frac{E a}{R T}
$$

Plotting $\ln (k)$ versus $(1 / T)$ for the temperature range at which the reaction occurs, gives a straight line, the slope of which is $-E a / R$ and the intercept is $\ln (A)$. This method is nevertheless limited to the determination of Arrhenius parameters for a single (or global) reaction: the case of simultaneous reactions cannot be treated, and this is done through a numerical procedure.

\subsection{Numerical method}

The second method is based on numerical parameter estimation. This approach, and the related difficulties, have been largely commented before [12,15]. It follows the following steps. For a given reaction scheme, the equations describing the mass evolution versus time (or temperature for ramp tests) can be written and solved for particular initial conditions and a given set of the Arrhenius parameters and stoechiometric coefficients. A calculation by an inverse method is then used to determine both the optimal Arrhenius parameters and the stoichiometric coefficients: the objective is to minimize the difference between the experimental mass 
evolution and the model predicted mass evolution. The fitting can be realized either on the mass evolution [1-3] itself or on the mass derivative evolution [6]. In this paper, TG curves have been used for the minimization. Details of the procedure are given below.

For each of the six schemes, we have written the ordinary differential equations (ODE) system linking the mass derivative of each solid species to the current mass of each solid (cardboard, Intermediate 1 and 2, char). For example, the equations obtained for scheme \#1 are the following [14]:

$$
\begin{aligned}
& \frac{\mathrm{d} m_{\mathrm{C}}(t)}{\mathrm{d} t}=-k_{1} m_{\mathrm{C}}(t)=-A_{1} \exp \left(-\frac{E a_{1}}{R T}\right) m_{\mathrm{C}}(t) \\
& \frac{\mathrm{d} m_{\mathrm{I} 1}(t)}{\mathrm{d} t}=a k_{1} m_{\mathrm{C}}(t)-a k_{2} m_{\mathrm{I} 1}(t) \\
& =a\left[A_{1} \exp \left(-\frac{E a_{1}}{R T}\right) m_{\mathrm{C}}(t)-A_{2} \exp \left(-\frac{E a_{2}}{R T}\right) m_{\mathrm{I} 1}(t)\right] \\
& \frac{\mathrm{d} m_{\mathrm{Char}}(t)}{\mathrm{d} t}=b k_{2} m_{\mathrm{I} 1}(t)=b A_{2} \exp \left(-\frac{E a_{2}}{R T}\right) m_{\mathrm{I} 1}(t)
\end{aligned}
$$

For convenience, we re-write these equations by introducing the temperature as the independent variable since the experiments are performed at a constant heating rate $\beta$. We have

$$
\begin{aligned}
& \frac{\mathrm{d} m_{\mathrm{C}}(T)}{\mathrm{d} T}=-\frac{1}{\beta} k_{1} m_{\mathrm{C}}(T)=-\frac{1}{\beta} A_{1} \exp \left(-\frac{E a_{1}}{R T}\right) m_{\mathrm{C}}(T) \\
& \begin{aligned}
\frac{\mathrm{d} m_{\mathrm{I} 1}(T)}{\mathrm{d} T} & =\frac{1}{\beta} a k_{1} m_{\mathrm{C}}(T)-\frac{1}{\beta} a k_{2} m_{\mathrm{I} 1}(T) \\
& =\frac{1}{\beta} a\left[A_{1} \exp \left(-\frac{E a_{1}}{R T}\right) m_{\mathrm{C}}(T)-A_{2} \exp \left(-\frac{E a_{2}}{R T}\right) m_{\mathrm{I} 1}(T)\right] \\
\frac{\mathrm{d} m_{\mathrm{Char}}(T)}{\mathrm{d} T} & =\frac{1}{\beta} b k_{2} m_{\mathrm{I} 1}(T)=\frac{1}{\beta} b A_{2} \exp \left(-\frac{E a_{2}}{R T}\right) m_{\mathrm{I} 1}(T)
\end{aligned}
\end{aligned}
$$

The process for computing the estimated total mass is the following:

(1) The ODE system corresponding to the chosen scheme is integrated. The numerical solver used is based on backward differentiation formulas, also called Gear's method, well suited for stiff problems. The Arrhenius parameters and stoechiometric coefficients (searched parameters) have to be fixed for the first iteration. For Scheme \#5, the proportion between $\mathrm{C} 1$ and $\mathrm{C} 2$ was an additional searched parameter that was fitted together with the Arrhenius parameters.

(2) The global mass is then computed by summing all the solid constituent masses, and the objective function $O F$ to be minimized is evaluated. This objective function consists in the least square differences between the experimental actual TG masses and the computed ones, i.e. we have 


$$
O F=\frac{1}{i j} \sum_{i} \sum_{j}\left(m_{\mathrm{tot}_{i j}}-\hat{m}_{\mathrm{tot}_{i j}}\right)^{2}
$$

where $m_{\text {tot }}$ is the total experimental mass sample normalized to the dry mass, $\hat{m}_{\text {tot }}$ is the model predicted normalized total mass, $j$ is a point on curve $i, i$ is the curve number corresponding to each experiment (i.e. experiment with a different heating rate).

(3) The classical Nelder Mead 'simplex' method is used to find the new values of the searched parameters. The 'simplex' method is a direct method, which presents the main advantage to avoid computing the Jacobian matrix. With the new values of the parameters, this process is repeated until convergence is achieved.

We have seen in the introduction that previous authors have suggested the introduction of non-constant Arrhenius parameters to take into account the influence of the heating rate. Such varying parameters are apparent parameters hiding more complex chemical mechanisms and/or mass and heat transfer phenomena. Generally, such results (non-constant kinetic parameters) can be used to provide Arrhenius parameters as functions of the heating rate through some nonlinear interpolation procedure. For practical reasons, it could be interesting to avoid such a complex non-linear model (not to consider the questions being raised about the physical significance of such non-constant values). To this purpose we propose in this paper to adopt the following procedure:

i) the Arrhenius parameters and stoechiometric coefficients are assumed to be constant, independent of the heating rate;

ii) the optimal parameters set is determined by taking all three experimental data in the objective function.

For all these computations, we have adopted the following conditions. Mass is normalized on the dry basis. Since the drying period finishes at about $450 \mathrm{~K}$, the experimental mass at this temperature is the initial mass taken into account in the normalization. The optimization was performed in the temperature range 450-873 $\mathrm{K}$. In order to fit several curves at the same time, it was necessary to re-sampleperiodically, with a constant step - the experimental data in order to get the same number of data points for each experiment.

The six reaction schemes described in Table 1 were tested. A typical optimization sequence lasted for $2 \mathrm{~h}$ on a Sun Ultra 10 Workstation, corresponding to approximately 1000 iterations. The resulting values of the objective function are given in Table 2, as well as the identified parameters. The first general observation that can be made is that, for all the tested reaction schemes, the Arrhenius parameters found for the first reaction are similar. This indicates that this apparent mechanism has a more chemically sounded basis. On the contrary, the parameters for the reactions following this first stage (second and third) are very different from one scheme to another.

The objective function $O F$ is the lowest for scheme \#6 and for scheme \#2. Nevertheless, plotting the reaction rate $k$ versus $1 / T$ for these two schemes revealed 


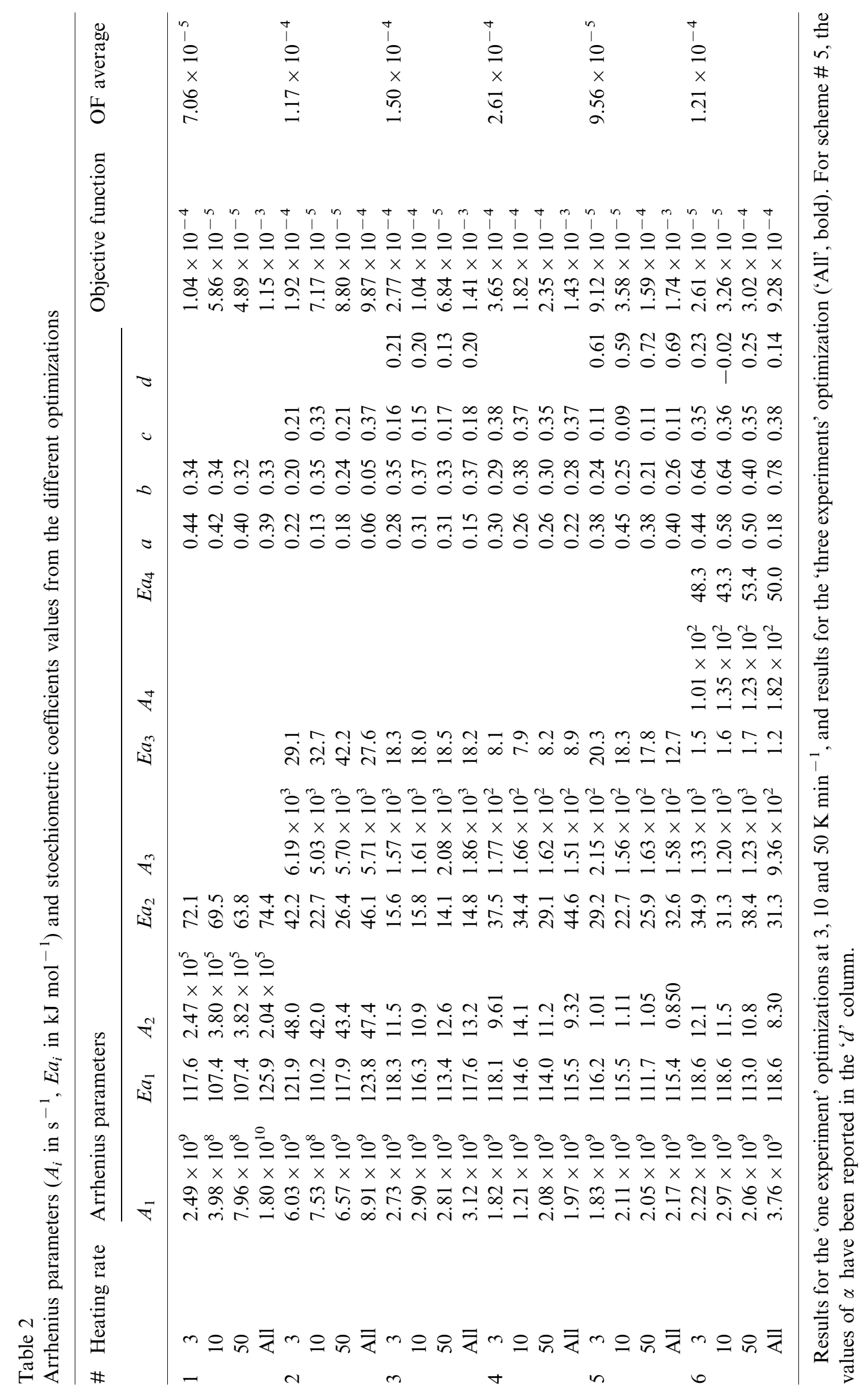


discontinuities in the curve, the values of $k$ tending towards zero at some points. Because of this, these two reaction schemes were considered as not acceptable.

The first acceptable result was reached with scheme \#1, whose $O F$ is just slightly higher than the one for schemes \#6 and \#2. In Fig. 4, we plot the reaction rates obtained with this scheme (a limited number of experimental data points is plotted for readability). They do not show any discontinuity. Even if there is a significant difference between the experimental and the model predicted reactions rates, this model can describe the differences in the $k$-values observed for the second pyrolysis stage during the experiments at different heating rates. In addition, the change in the slope between the two pyrolysis stages is recovered, despite the fact that the change is not as marked as during the experiments.

We have plotted in Fig. 5 the experimental and model predicted mass evolutions versus temperature, which is another way to view the fitting performance. Some differences can be observed, and this shows that a very accurate description is not possible by using such simple schemes. For most practical cases, however, the error made will be considered as acceptable, and might be lower than the error introduced by the classical method in which a non-linear interpolation of Arrhenius parameters is done, as discussed below. If the accuracy is acceptable, this model must be used preferably since it is very practical for modeling purposes, because the kinetic parameters are known constants, independent of the heating rate.

It is indeed relevant to examine the results for the Arrhenius parameters and stoechiometric coefficients when they are estimated three times for each experiment, using a single experiment each time. The results are also reported in Table 2. The best average score for the $O F$ is now reached by scheme \#1. As illustrated in Fig. 6, good

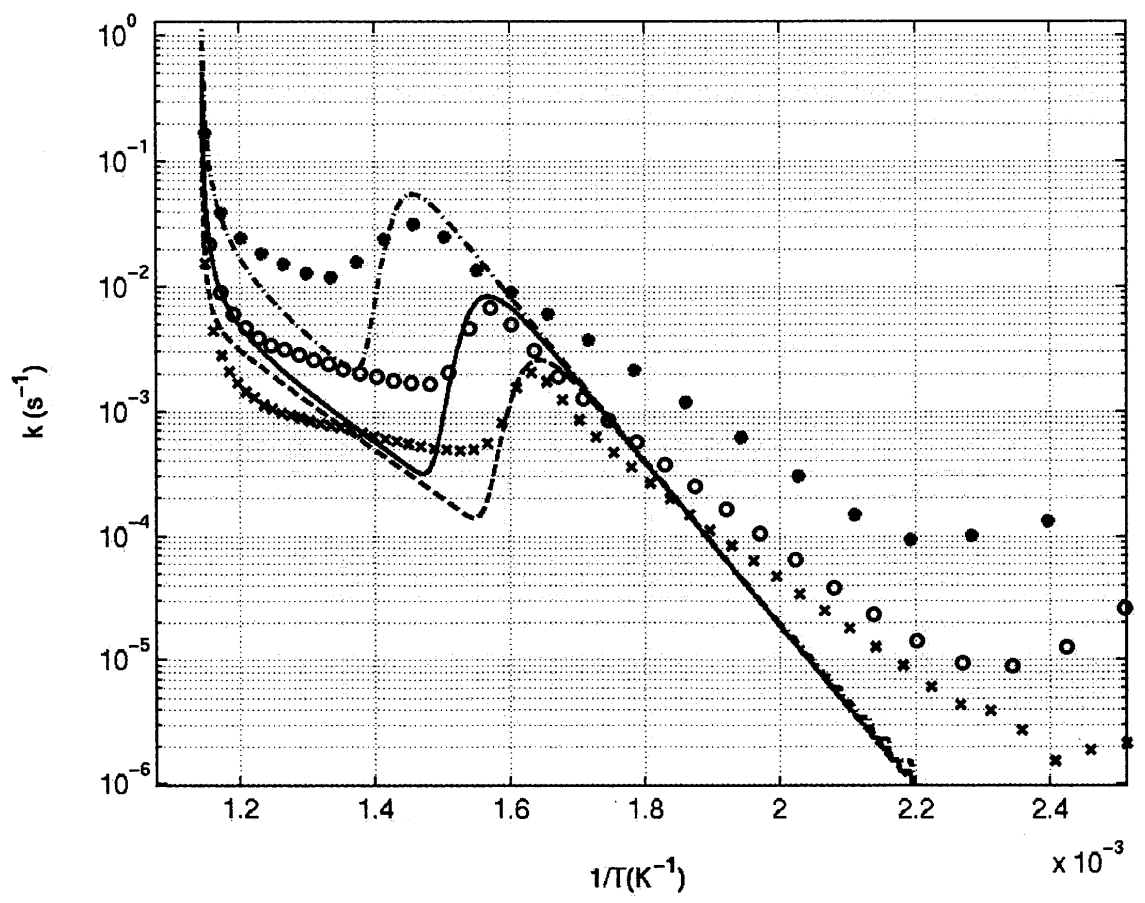

Fig. 4. Comparison between the experimental reactions rates at $(\times) 3 \mathrm{~K} \mathrm{~min}^{-1}$, (○) $10 \mathrm{~K} \mathrm{~min}^{-1},(\bullet) 50$ $\mathrm{K} \min ^{-1}$, and the modeled reactions rates at (- $) 3 \mathrm{~K} \mathrm{~min}^{-1}$, (-) $10 \mathrm{~K} \mathrm{~min}^{-1}$ and (- .) $50 \mathrm{~K} \mathrm{~min}^{-1}$. 


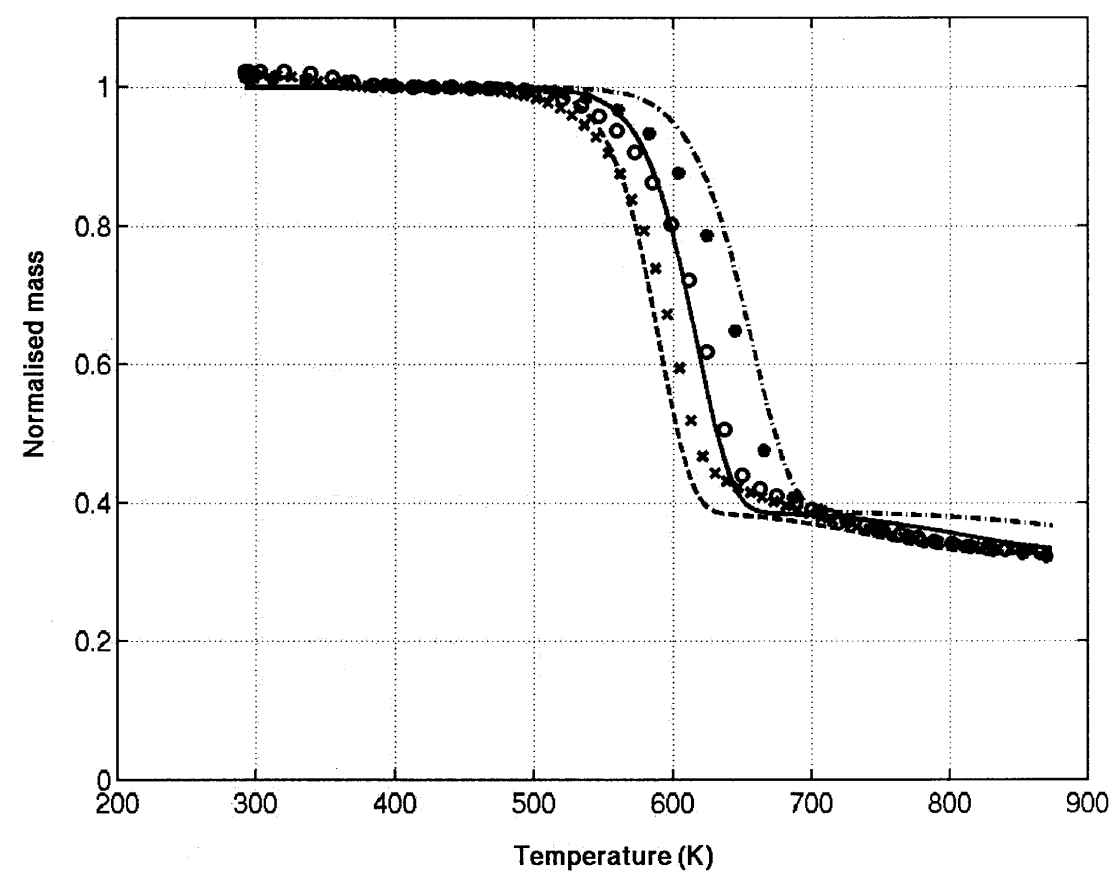

Fig. 5. Comparison between the experimental normalized mass evolution at $(\times) 3 \mathrm{~K} \mathrm{~min}^{-1}$, (O) $10 \mathrm{~K}$ $\min ^{-1}$, (○) $50 \mathrm{~K} \mathrm{~min}^{-1}$, and the modeled normalized mass evolution at (- -) $3 \mathrm{~K} \mathrm{~min}^{-1}$, (-) $10 \mathrm{~K} \mathrm{~min}^{-1}$ and (- .) $50 \mathrm{~K} \mathrm{~min}^{-1}$ with a single parameter set for the three experiments.

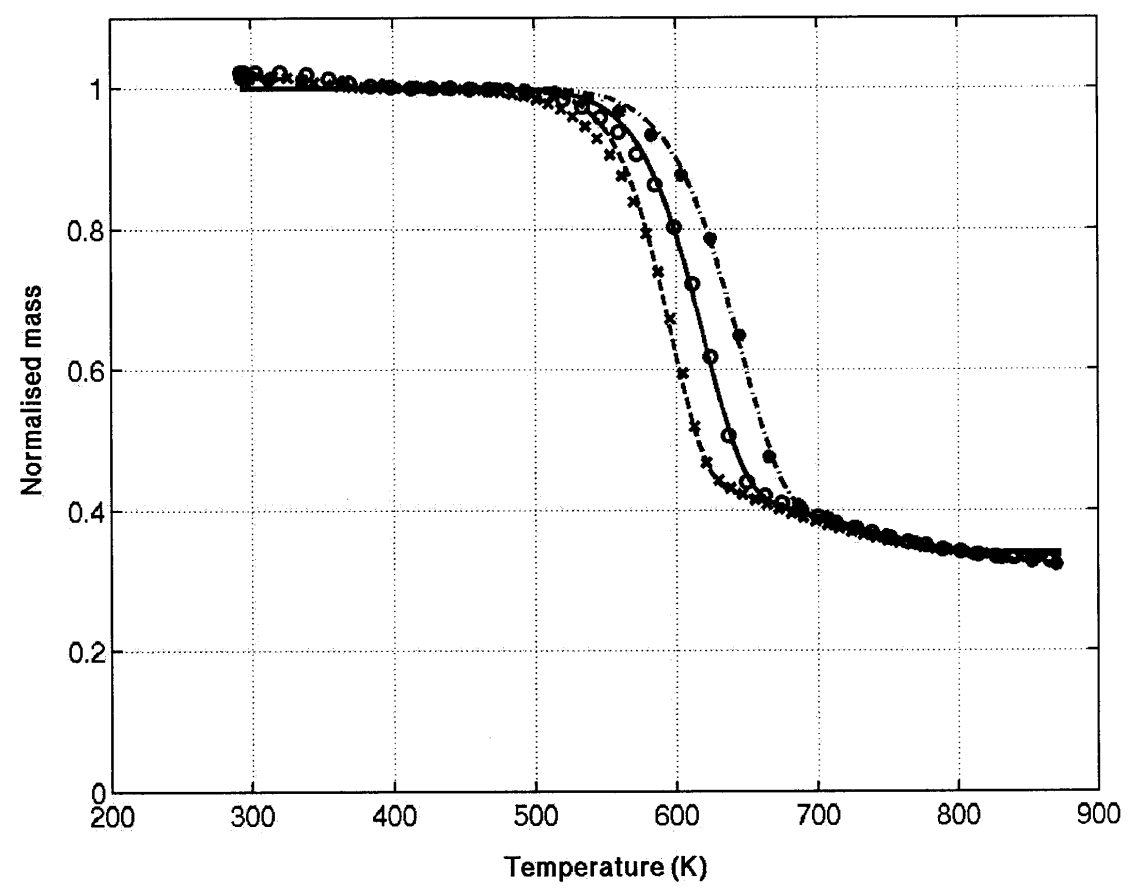

Fig. 6. Comparison of the experimental normalized mass evolution at ( $\times$ ) $3 \mathrm{~K} \mathrm{~min}^{-1}$, ( $\left.\bigcirc\right) 10 \mathrm{~K}^{-1} \mathrm{~min}^{-1}$, (O) $50 \mathrm{~K} \mathrm{~min}^{-1}$, and the modeled normalized mass evolution at (- -) $3 \mathrm{~K} \mathrm{~min}^{-1}$, (-) $10 \mathrm{~K} \mathrm{~min}^{-1}$ and (- .) $50 \mathrm{~K} \mathrm{~min}^{-1}$, with a different set of parameters for each experiment. 


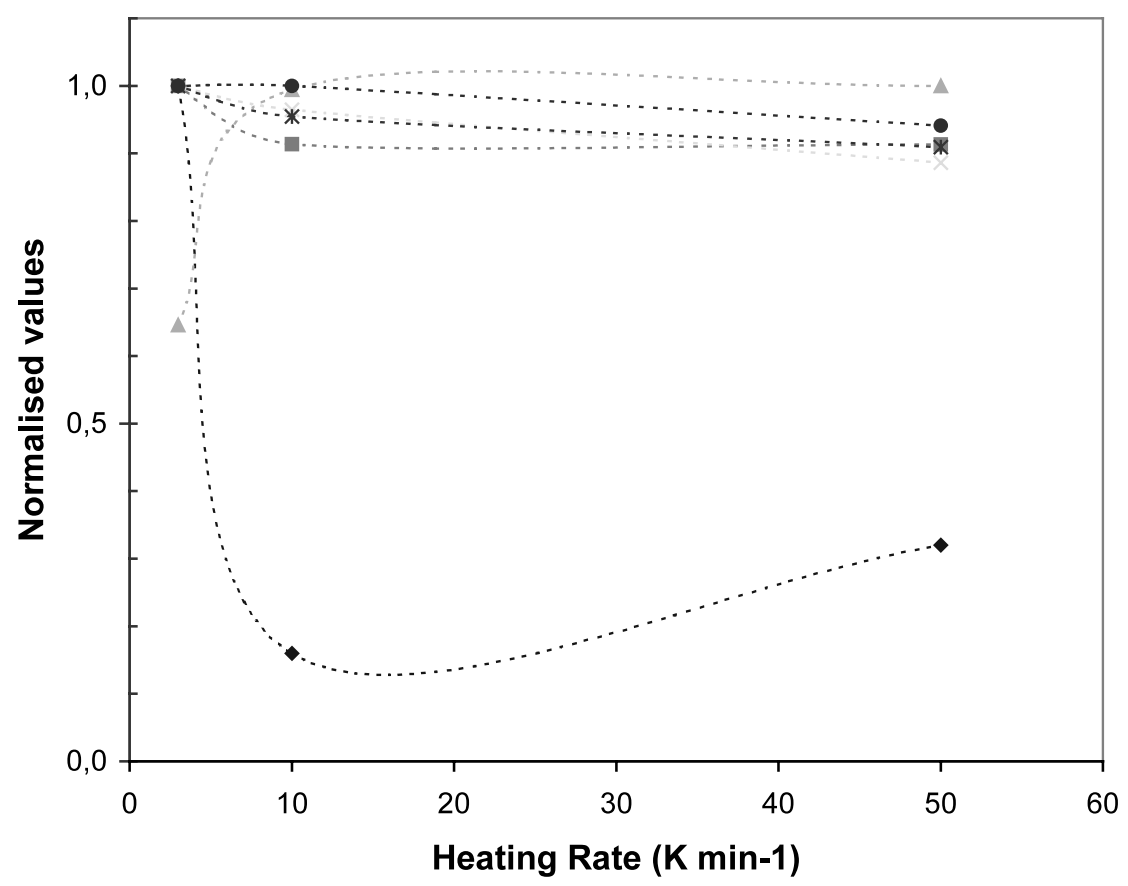

Fig. 7. Evolution of the Arrhenius parameters and the stoechiometric coefficients with the heating rate. (४) $A_{1} ;(\mathbf{\square}) E a_{1} ;(\boldsymbol{\Delta}) A_{2} ;(\times) E a_{2} ;(*) a ;(\mathbf{O}) b$.

fits are now obtained for each experiment. The Arrhenius parameters on one hand and the stoechiometric coefficients on the other hand are different from one fit at a given HR to another fit at a different HR. This is illustrated in Fig. 7 which represents the Arrhenius parameters and the stoichiometric coefficients versus HR. No clear tendency is observed: some of the parameters, such as $b$, are the same for the tests at 3 and $10 \mathrm{~K} \mathrm{~min}^{-1}$, but change between the tests at 10 and $50 \mathrm{~K} \mathrm{~min}^{-1}$. Some of the parameters, such as $E a_{1}$ and $A_{2}$, change between the tests at 3 and $10 \mathrm{~K}$ $\min ^{-1}$ but remain the same between the tests at 10 and $50 \mathrm{~K} \mathrm{~min}^{-1}$, when others (such as $E a_{2}$ and $a$ ) give a different value for each HR. At this point, the difficulty that will be encountered when establishing the interpolation function for these parameters values must be emphasized. As shown in Fig. 7, their evolution is not linear, and it is very delicate to fix an interpolation curve at this point without further analysis. This justifies the attempt made in this paper at developing a more robust - while perhaps less accurate — scheme that could be used in a wide range of operating conditions.

For most of the reaction schemes, the $O F$ for the fit performed on the three experiments together is much higher-typically 10 times - than the highest $O F$ obtained for the three separate fits. One could conclude from the fits on single experiments that scheme \#1 (for example) does somewhat describe the chemical reaction sequence involved in the thermal degradation of cardboard. However, if it would be the case, the fitted coefficients should not depend upon the heating rate. Therefore, one must be cautious about the interpretation of such good fits.

One can also note that, in the case of scheme $\# 6$ with $\mathrm{HR}=10 \mathrm{~K} \mathrm{~min}^{-1}$, a negative value is identified for the stoechiometric coefficient $d$, which is not 
physically acceptable. This could mean that the algorithm has converged towards one of the local minima of the optimization problem, consisting in the minimization of the objective function defined in Eq. (11). The convergence towards the global minimum for each set of parameter is never certified. The practical solution in order to avoid the convergence towards a local minimum consists in launching the optimization procedure from different starting values and comparing the obtained solutions. In our case, the optimization procedure performed with different starting values always converged towards a negative value of $d$. It is not clear what are the mathematical implications of this behavior. In any case, this suggests that the use of this scheme, with the available data, is not recommended because of this lack of robustness.

\section{Conclusion}

TGA can provide precise and reproducible mass loss curves for cardboard pyrolysis under heating rates between 3 and $50 \mathrm{~K} \mathrm{~min}^{-1}$, which is relevant for the determination of a reaction scheme of interest for practical applications. Such a scheme can be used in a model that solves heat and mass transfer equations for the prediction of local reaction rates inside large solid bodies submitted to pyrolysis or combustion.

The analysis of the reaction rates observed under heating rates of 3,10 and $50 \mathrm{~K}$ $\mathrm{min}^{-1}$ indicates a complex sequence of reactions. A ratio of up to 20 in the values of reactivity for a given temperature is observed between the experiments at different heating rates.

Our attempt to determine a pseudo-species reaction scheme involving several reactions and a single set of Arrhenius parameters and stoechiometric coefficients to describe the thermal degradation of cardboard has lead to several observations:

- As far as the parameter estimation method is concerned, some difficulties may arise due to the determination of physically non-acceptable reaction schemes (negative stoechiometric coefficients and discontinuities in the reaction rate values). While the proposed reaction schemes are consistent in terms of chemistry, the origin of the obtained non-physical parameters can be only explained by the convergence towards local minima, as explained above. New optimization algorithms, such as optimization with constraints, will be used in the future in order see if such problems may be avoided.

- The different tested reaction schemes do not lead to very different $O F$. Therefore, it is not possible to infer from this analysis the most representative reaction scheme in terms of the chemical mechanisms involved.

- Predictions with a very good accuracy were not reached, i.e. accuracy close to the experimental errors. A practical reaction scheme is nevertheless proposed, that can be used to simulate the mass loss rate of large solid bodies under typical industrial conditions of pyrolysis or combustion. The set of kinetics parameters proposed has the advantage to be independent of the heating rate. 
The proposed reaction scheme (Scheme \#1) and its related parameters can be summarized as

$$
\begin{aligned}
& C \stackrel{k_{1}}{\rightarrow} a \mathrm{I} 1+a^{\prime} G \quad a=0.3871 \quad b=0.3258 \\
& a \mathrm{I} 1 \stackrel{k_{2}}{\rightarrow} b \mathrm{Char}+b^{\prime} G \\
& \frac{\mathrm{d} m_{\mathrm{C}}(t)}{\mathrm{d} t}=-A_{1} \exp \left(-\frac{E a_{1}}{R T}\right) m_{\mathrm{C}}(t) \quad A_{1}=1.81 \times 10^{10} \mathrm{~s}^{-1} \quad E a_{1} \\
& =125850 \mathrm{~J} \mathrm{~mol}^{-1} \\
& \frac{\mathrm{d} m_{\mathrm{I} 1}(t)}{\mathrm{d} t}=a\left[A_{1} \exp \left(-\frac{E a_{1}}{R T}\right) m_{\mathrm{C}}(t)-A_{2} \exp \left(-\frac{E a_{2}}{R T}\right) m_{\mathrm{I} 1}(t)\right] \quad A_{2} \\
& =20374 \mathrm{~s}^{-1} \quad E a_{2}=74422 \mathrm{~J} \mathrm{~mol}^{-1} \\
& \frac{\mathrm{d} m_{\mathrm{Char}}(t)}{\mathrm{d} t}=b A_{2} \exp \left(-\frac{E a_{2}}{R T}\right) m_{I 1}(t)
\end{aligned}
$$

When the parameters estimation is performed on experiments at different heating rates taken separately, much better fits are obtained. However, it should not be concluded from such tests that the reaction scheme describes correctly, from a physical point of view, the thermal degradation.

The scheme leading to the best average fit is Scheme \#1. Table 2 gives the Arrhenius parameters and stoechiometric coefficients to be used at the three heating rates. We remind the reader that this formulation raises the problem of the nonlinear interpolation of the parameters that has to be operated when their values are needed at any heating rate.

This parameter determination method can be applied to the study of the reaction schemes for the thermal decomposition of other materials than cardboard. This work confirms the necessity to have more information than a mass evolution in order to derive a chemically well-understood mechanism. Gas analysis from coupled TGFTIR or TG-MS is probably one of the best candidate for this further development.

\section{References}

[1] A.K. Gupta, J. Propulsion Power 15 (2) (1999) 187.

[2] C.H. Wu, C.Y. Chang, J.P.J. Lin, Chem. Tech. Biotech. 68 (1997) 65.

[3] R. Alén, E. Kuoppala, P. Oesch, J. Anal. Appl. Pyrol. 36 (1996) 137-148.

[4] M.R. Hajaligol, J.B. Howard, J.P. Longwell, W.A. Peters, Ind. Eng. Chem. Proc. Des. Dev. 21 (1982) 457.

[5] M.R. Hajaligol, W.A. Peters, J.B. Howard, Symp. Fund. Res. in Heat and Mass Transfer in Two and Three Phase System, AICHE, Washigton DC, 1988.

[6] A. Missoum, A.K. Gupta, J. Chen, E.L. Keating, ASME Computers in Engineering Conference, California, 1996. 
[7] M.J. Antal, G. Varhegyi, Ind. Eng. Chem. Res. 34 (1995) 703.

[8] I. Milosavljevic, J. Oja, E.M. Suuberg, Ind. Eng. Chem. Res. 35 (1996) 653.

[9] I. Milosavljevic, E.M. Suuberg, Ind. Eng. Chem. Res. 34 (4) (1995) 1081.

[10] A. Broido, M.A. Nelson, Comb. Flame 24 (1975) 263.

[11] A.G.K. Bradbury, Y. Sakai, F. Shafizadeh, J. Appl. Polym. Sci. 23 (1979) 3271.

[12] R. Font, I. Martin-Gullon, M. Esperenza, A. Fullana, J. Anal. Appl. Pyrol. $58-59$ (2001) 703.

[13] J. Reina, E. Velo, L. Puigjaner, Ind. Eng. Chem. Res. 37 (11) (1998) 4290.

[14] I. Martin-Gullon, M. Esperenza, R. Font, J. Anal. Appl. Pyrol. 58-59 (2001) 635.

[15] J.A. Conesa, A. Marcilla, J.A. Caballero, J. Anal. Appl. Pyrol. 58-59 (2001) 617. 